\title{
Thermo-Diffusion and Diffusion -Thermo Effects on Convective Heat and Mass Transfer through a Porous Medium in a Circular Cylindrical Annulus with Quadratic Density Temperature Variation - Finite Element Study
}

\author{
P. S. Reddy ${ }^{1+}$, V. P. Rao ${ }^{2}$ \\ ${ }^{1}$ Asst. Professor, Dept of Mathematics, RGM Engg. College, Nandyal, JNTU Anantapur, India \\ ${ }^{2}$ Sr. Professor, Dept of Mathematics,SK University, Anantapur, India \\ †Corresponding Author Email:suda1983@gmail.com
}

(Received June 12, 2010; accepted July 12, 2011)

\begin{abstract}
In this paper the heat and mass transfer characteristics of mixed convection about a circular cylindrical annulus in a porous medium, by taking into account the Thermo-Diffusion (Soret) and Diffusion -Thermo (Dufour) effects have been analyzed. The governing partial differential equations are transformed into a set of coupled ordinary differential equations, which are evaluated numerically by using a finite element method. The velocity, temperature and concentration profiles are presented graphically for various values of the parameters entering in to the problem. The Nusslet number, Sherwood number and Shear stress are summarized in tabular form.
\end{abstract}

Keywords: Heat transfer, Mass transfer, Soret effect, Dufour effect, Quadratic density temperature variation and finite element method.

\section{INTRODUCTION}

The flow of an incompressible, viscous fluid through a porous annulus has drawn the attention of several authors in the last three decades in view of its technological applications (Berman 1958; Ram et al. 1977; Kapoor et al. 1960; Mahapatra 1973). It is well known that some liquids attain their maximum density only at a particular temperature range. At such temperature range the equation of state which relates the density and temperature may not be in the usual linear form. In such case a non-linear density relation may be best suited to describe the physical phenomenon. For example, in dealing with flow of water at $4^{\circ} \mathrm{C}$ it has been observed that Goren, S.L. (1966) a quadratic density temperature variation (QDT) gives a better understanding of the phenomenon in comparison to the usual linear relation (LDT). Keeping this in view a few authors have discussed the hydrodynamic convection flows in different configurations using a non-linear density temperature variation. This has been extended to hydro magnetic by Sarojamma (1981) and Sivaprasad. A few of the investigations include the effect of the additional heat source which is either constant or temperature dependent (Agaral et al. 1976; Bhargava et al. 1979; Cunningham et al. 1980; Gilpin 1975; Roy 1972). Reddy (2009) has analyzed the convective heat and mass transfer flow with thermo-diffusion effects in cylindrical annulus. Recently Padmavathi (2009) has studied the finite element analysis of the convective heat transfer through a cylindrical annulus with quadratic temperature variation.

Coupled heat and mass transfer driven by natural convection in a fluid saturated porous medium has considerable interest in recent years, due to many important applications in engineering and geophysical applications. As many industrially and environmentally relevant fluids are not pure, it is been suggested that more attention should be paid to convective phenomena which can occur in mixtures, but are not in common liquids such as air or water. Applications involving liquid mixtures include the costing of alloys, ground water pollutant migration and separation operations. In all of these situations, multi component liquids can undergo natural convection driven by buoyancy force resulting from simultaneous temperature and species gradients. In the case of binary mixtures, the species gradients can be established by the applied boundary conditions such as species rejection associated with alloys costing, or can be induced by transport mechanism such as Soret (thermo) diffusion. In the case of Soret diffusion, species gradients are established in an otherwise uniform concentration mixture in accordance with Onsager reciprocal relationship. Thermal-diffusion known as the Soret effect takes place and as a result a mass fraction distribution is established in the liquid layer. The sense of migration of the molecular species is determined by the sign of Soret coefficient. The Soret effect for instance, has been 
utilized for isotope separation and in mixtures between gases with very low molecular weight $\mathrm{H}_{2}$ or $\mathrm{He}$ and the medium molecular weight $\mathrm{N}_{2}$ or air.

In this paper we discuss the free and forced convection flow through a porous medium in a circular cylindrical annulus with Thermal-Diffusion and Diffusion-Thermo effects in the presence of Quadratic Temperature Density variation, where the inner wall is maintained constant temperature while the outer wall is maintained constant heat flux and the concentration is constant on the both walls. By using Galerkin finite element analysis the coupled momentum, energy and diffusion equations are solved.

\section{Formulation of the Problem}

We consider free and forced convection flow of a viscous fluid in a circular cylindrical annulus with Thermal-Diffusion and Diffusion-Thermo effects in the presence of Quadratic Temperature Density variation, where the inner wall is maintained constant temperature while the outer wall is maintained constant heat flux and the concentration is constant on the both walls. Both the fluid and porous region have constant physical properties and the flow is a mixed convection flow taking place under thermal buoyancy and molecular buoyancy, uniform axial pressure gradient. The Brinkman-Forchheimer-Extended Darcy model which accounts for the inertia and boundary effects has been used for the momentum equation in the porous region. We take quadratic density variation in the equation of state. Here, the thermo physical properties of the solid and fluid have been assumed to be constant except for the density variation in the body force term (Boussinesque approximation), and the solid particles and fluid are considered to be in local thermal equilibrium. Since the flow is unidirectional, the equation of continuity reduces to $\frac{\partial u}{\partial z}=0$, where ' $u$ ' is the axial velocity implies $u=u(r)$. Also the flow is unidirectional along the axial cylindrical annulus. Making use of the above assumptions the governing equations are

Equation of linear momentum

$-\frac{\partial p}{\partial z}+\frac{\mu}{\delta}\left(\frac{\partial^{2} u}{\partial r^{2}}+\frac{1}{r} \frac{\partial u}{\partial r}\right)-\frac{\mu}{k} u-\frac{\rho \delta F}{\sqrt{k}} u^{2}+$

$\rho g \beta\left(T-T_{0}\right)^{2}-\rho_{0} \beta * g\left(C-C_{0}\right)=0$

Equation of Energy

$\rho c_{p} u \frac{\partial T}{\partial z}=\lambda\left(\frac{\partial^{2} T}{\partial r^{2}}+\frac{1}{r} \frac{\partial T}{\partial r}\right)+\frac{D_{m} K_{t}}{c_{s} c_{p}}\left(\frac{\partial^{2} c}{\partial r^{2}}+\frac{1}{r} \frac{\partial c}{\partial r}\right)$

Equation of Diffusion

$u \frac{\partial C}{\partial z}=D_{1}\left(\frac{\partial^{2} C}{\partial r^{2}}+\frac{1}{r} \frac{\partial C}{\partial r}\right)+\frac{D_{m} K_{t}}{T_{m}}\left(\frac{\partial^{2} T}{\partial r^{2}}+\frac{1}{r} \frac{\partial T}{\partial r}\right)$

Equation of stat

$\rho-\rho_{0}=-\beta \rho_{0}\left(T-T_{0}\right)^{2}-\beta^{*} \rho_{0}\left(C-C_{0}\right)$

Where $u$ is the axial velocity in the porous region, $T \&$ $\mathrm{C}$ are the temperature and concentrations of the fluid, $\mathrm{k}$ is the permeability of porous medium, $\mathrm{F}$ is a function that depends on Reynolds number and the microstructure of the porous medium and $D_{l}$ is the Molecular diffusivity , $D_{m}$ is the coefficient of mass diffusitivity, $T_{m}$ is the mean fluid temperature, $\mathrm{K}_{\mathrm{t}}$ is the thermal diffusion, $C_{s}$ is the concentration susceptibility, $C_{p}$ is the specific heat, $\rho$ is density, $g$ is gravity, $\beta$ is the coefficient of thermal expansion, $\beta^{*}$ is the coefficient of volume expansion

The boundary conditions relevant to

$$
\begin{aligned}
& u=0 \quad \& \quad T=T_{i}, \quad C=C_{i} \quad \text { at } \quad r=a \\
& u=0 \quad \& \quad \frac{\partial T}{\partial r}=Q_{1}, C=C_{0}, \quad \text { at } \quad r=a+s
\end{aligned}
$$

The axial temperature gradient $D u=\left(\frac{D_{m} K_{t} \Delta c a^{2}}{C_{s} C_{p} \Delta T \lambda}\right)$ and concentration gradient $\frac{\partial C}{\partial z}$ are assumed to be constant say A and B respectively.

We now define the following non-dimensional variables

$$
\begin{aligned}
& z^{*}=\frac{z}{a}, \quad r^{*}=\frac{r}{a}, \quad u^{*}=\frac{a}{v} u, \quad s^{*}=\frac{s}{a} \\
& p^{*}=\frac{p a \delta}{\rho v^{2}} \quad, \theta^{*}=\frac{T-T_{i}}{A a} \quad, C^{*}=\frac{C-C_{i}}{C_{i}-C_{0}},
\end{aligned}
$$

Using Eqs. (2.5) \& (2.6), Eqs. (2.2) \& (2.3) reduces to

$$
\begin{aligned}
& \rho_{0} C_{p} u A=\lambda\left(T_{r r}+\frac{1}{r} T_{r}\right)+\frac{D_{m} K_{t}}{C_{s} C_{p}}\left(C_{r r}+\frac{1}{r} C_{r}\right) \\
& \rho_{0} C_{p} u B=\lambda\left(C_{r r}+\frac{1}{r} C_{r}\right)+\frac{D_{m} K_{t}}{C_{s} C_{p}}\left(T_{r r}+\frac{1}{r} T_{r}\right)
\end{aligned}
$$

$\frac{d^{2} u}{d r^{2}}+\frac{1}{r} \frac{d u}{d r}=P+\delta\left(D^{-1}\right) u+\delta^{2} \Lambda u^{2}-\delta G \theta^{2}$

$\frac{d^{2} \theta}{d r^{2}}+\frac{1}{r} \frac{d \theta}{d r}=P_{r} N_{t} u+D u N t\left(\frac{d^{2} C}{d r^{2}}+\frac{1}{r} \frac{d C}{d r}\right)$

$\frac{d^{2} C}{d r^{2}}+\frac{1}{r} \frac{d C}{d r}=S c N C u+S c S r\left(\frac{d^{2} \theta}{d r^{2}}+\frac{1}{r} \frac{d \theta}{d r}\right)$

where

$\Lambda=F D^{-1}$

(Forchheimer number)

$P_{r}=\frac{\mu C_{p} v}{\lambda}$

(Prandtl number)

$G=\frac{g \beta\left(T_{1}-T_{0}\right) a^{3}}{v^{2}}$

(Grashof number)

$D^{-1}=\frac{a^{2}}{k}$

(Inverse Darcy parameter)

$N_{t}=\frac{A a}{T_{1}-T_{0}}$

(Temperature gradient) 
$D u=\left(\frac{D_{m} K_{t} \Delta c a^{2}}{C_{s} C_{p} \Delta T \lambda}\right)$

(Dufour Number)

$S c=\frac{v}{D_{1}}$

(Schmidt number)

$S r=\left(\frac{D_{m} K_{t} \Delta T}{v T_{m} \Delta C}\right)$

( Soret number)

$N_{c}=\frac{B a}{C_{1}-C_{0}}($ Non-dimensional concentration gradient $)$

The corresponding boundary conditions are

$u=0, \theta=0, \quad C=1, \quad$ at $\quad r=1$

$u=0, \frac{\partial \theta}{\partial r}=Q_{1}, C=0$, at $\quad r=1+s$

\section{ANALYSIS OF THE FLOW}

To solve Eqs. (2.9) to (2.11) together with the boundary conditions (2.12), we make use of the Galerkin finite element analysis with quadratic polynomial approximation functions. The velocity, temperature and concentration profiles has been discussed computationally for different variations in governing parameters by using Mathematica4.1.The Galerkin methods has been adopted in the variational formulation in each element to obtain the global coupled matrices for the velocity, temperature and concentration in course of the finite element analysis. The fundamental steps comprising the finite element method are now summarized:

Phase 1] Discretization of the domain into elements

Phase 2] Derivation of element equations

Phase 3] Assembly of Element Equations

Phase 4] Imposition of boundary conditions

Phase 5] Solution of assembled equations

The shear stress are evaluated on the cylinder using the formula

$\tau=\left(\frac{d u}{d r}\right)_{r=1,1+s}$

The rate of heat transfer (Nusselt number) are evaluated on the cylinder using the formula

$$
N u=-\left(\frac{d \theta}{d r}\right)_{r=1}
$$

The rate of mass transfer (Sherwood Number) is evaluated using the formula

$$
S h=-\left(\frac{d C}{d r}\right)_{r=1,1+s}
$$

\section{DisCUSSION OF THE NUMERICAL RESULTS}

In this analysis we investigate Thermo-Diffusion and Diffusion-Thermo effects on convective heat and mass transfer flow of a viscous fluid through a porous medium in concentric cylinders with quadratic temperature variation. The inner cylinder is maintained at constant temperature and the outer wall is maintained constant heat flux while the concentration is maintained constant on both the cylinders. The axial flow is in vertically downward direction, and hence the actual axial flow $\mathrm{u}$ is negative and hence $\mathrm{u}>0$ indicates a reversal flow. The velocity, temperature and concentration distributions are shown in Figs. 1 to 18 for different values of the parameters $G, D^{-1}, S c, S r, N$, and $D u$.

Figure 1 represents the variation of $\mathrm{u}$ with Grashof number $\mathrm{G}$. we notice that the actual axial flow enhances with increase in $G$. The variation of u with Darcy's parameter $D^{-1}$ shows that lesser the permeability of porous medium larger $|\mathrm{u}|$ everywhere in the flow region (Fig. 2). From Fig.3 we notice that lesser the molecular diffusitivity lager $|\mathrm{u}|$ in the flow region and attains maximum at $r=1.5$. From Fig. 4 we conclude that the variation of $\mathrm{u}$ with Soret parameter $\mathrm{Sr}$ experiences an enhancement in the flow region. The variation of $u$ with Dufour parameter $\mathrm{Du}$ shows that $|\mathrm{u}|$ experiences an enhancement with increase in $D u \leq 1$ and for further higher values of $D u \geq 1.3$ it depreciates (Fig. 6). The variation of $\mathrm{u}$ with buoyancy ratio parameter $\mathrm{N}$ shows that when the molecular buoyancy force dominates over the thermal buoyancy force $|\mathrm{u}|$ experiences an enhancement irrespective of the directions of the buoyancy forces (Fig. 5).

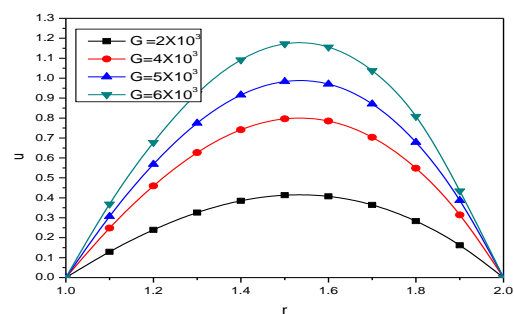

Fig.1. Variation of u with $G, D^{-1}=2 X 10^{3}, S c=1.3$, $\mathrm{Sr}=0.5, \mathrm{Du}=0.5, \mathrm{~N}=1$

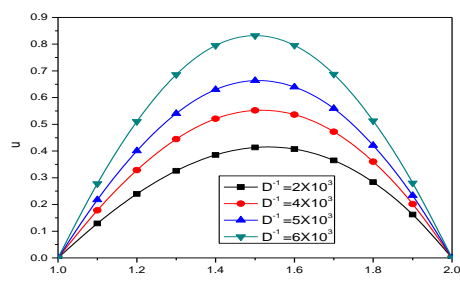

Fig.2. Variation of u with $D^{-1}, G=2 X 10^{3}, S c=1.3$, $\mathrm{Sr}=0.5, \mathrm{Du}=0.5, \mathrm{~N}=1$

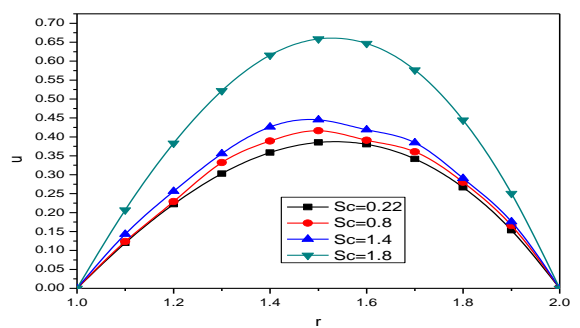

Fig.3. Variation of u with $S c, G=2 \times 10^{3}, D^{-1}=2 \times 10^{3}$, $\mathrm{Sr}=0.5, \mathrm{Du}=0.5, \mathrm{~N}=1$ 


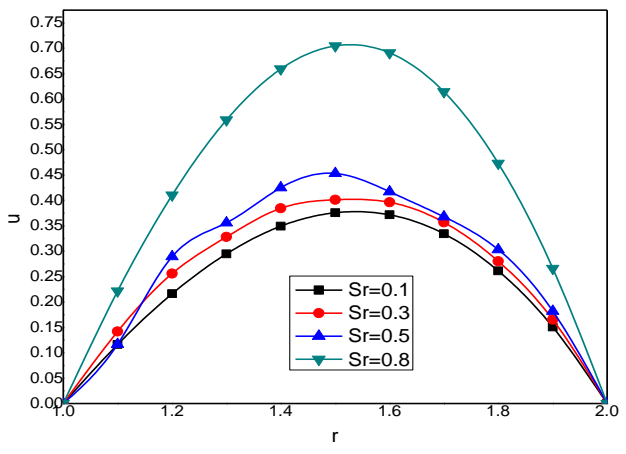

Fig.4. Variation of u with $S r, G=2 X 10^{3}, S c=1.3, D$ ${ }^{l}=2 X 10^{3}, D u=0.5, N=1$

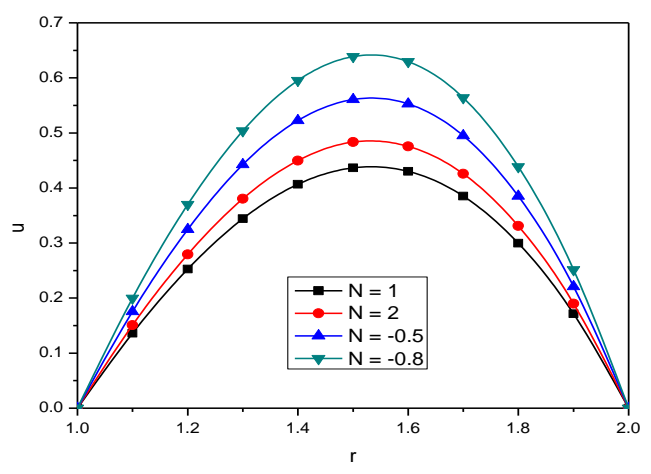

Fig.5. Variation of u with $N, G=2 X 10^{3}, S c=1.3, S r=0.5$, $D u=0.5, D^{-1}=2 X 10^{3}$

The non-dimensional temperature $(\theta)$ is shown in Figs. 7 to 12 for different values of the parameters $\mathrm{G}, \mathrm{D}^{-1}, \mathrm{Sc}$, $\mathrm{Sr}, \mathrm{N}$, and $\mathrm{Du}$. It is found that the non-dimensional temperature gradually increases from its prescribed value 0 on $r=1$ to attain its prescribed value 1 at $r=2$. The variation of $\theta$ with $G$ shows that an increase in $G$ depreciates $\theta$ in the flow region (Fig. 7). From Fig. 8 we conclude that lesser the permeability of porous medium smaller the actual temperature in the flow region. With respect to the variation of $\theta$ with $\mathrm{Sc}$, we notice that lesser the molecular diffusitivity lager the actual temperature in the flow region (Fig. 9).

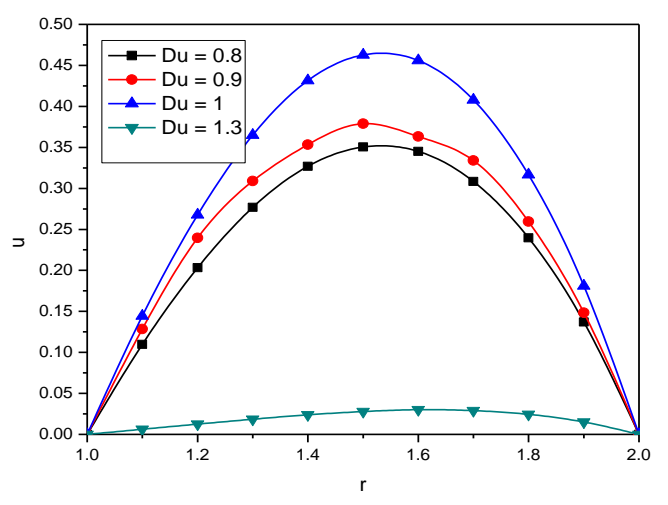

Fig.6. Variation of u with $D u, G=2 X 10^{3}, S c=1.3$, $\mathrm{Sr}=0.5, D^{-1}=2 \times 10^{3}, \mathrm{~N}=1$

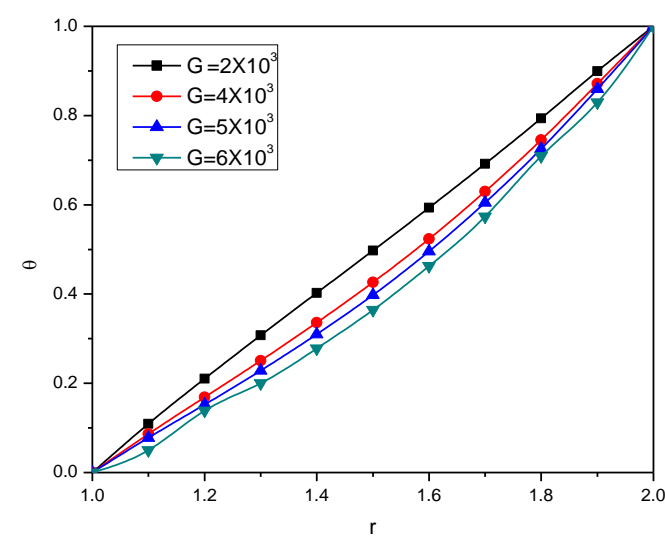

Fig.7. Variation of u with $G, D^{-1}=2 X 10^{3}, S c=1.3$, $S r=0.5, D u=0.5, N=1$

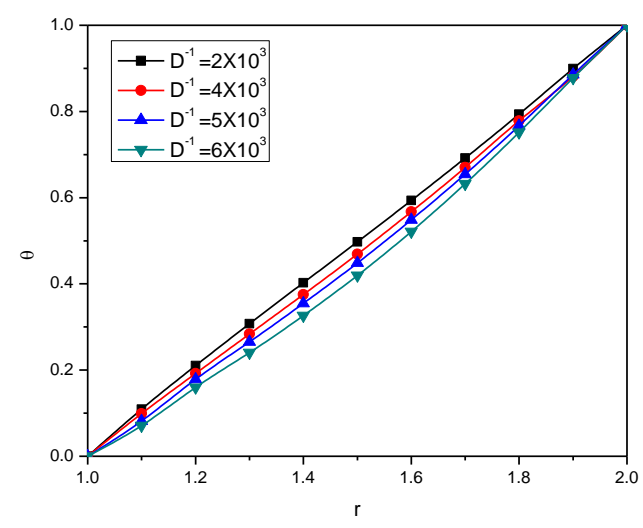

Fig.8. Variation of u with $D^{-1}, G=2 \times 10^{3}, S c=1.3$, $\mathrm{Sr}=0.5, \mathrm{Du}=0.5, \mathrm{~N}=1$

Figure 10 shows that the temperature experiences an enhancement in the flow region with $\mathrm{Sr}$ and it attains maximum at $r=1.8$. When the molecular buoyancy force dominates over the thermal buoyancy force it experiences depreciation irrespective of the directions of the buoyancy forces (Fig. 11). The variation of $\theta$ with Dufour parameter Du shows that the actual temperature experiences an enhancement in the flow region with Du (Fig. 12).

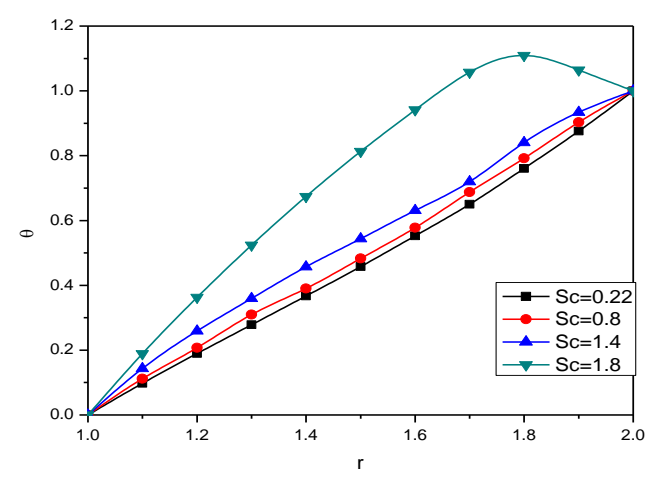

Fig.9. Variation of $\theta$ with $S c$., $G=2 X 103, D-1=2 X 103$, $S r=0.5, D u=0.5, N=1$ 


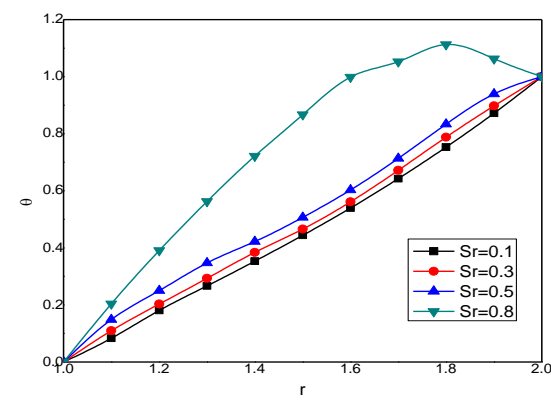

Fig.10. Variation of $\theta$ with $S r$., $G=2 X 103, S c=1.3, D$ $1=2 X 103, D u=0.5, N=1$

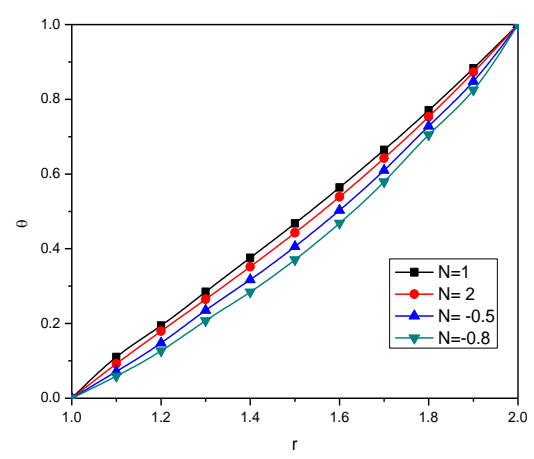

Fig.11. Variation of $\theta$ with $N, G=2 X 103, S c=1.3$, $S r=0.5, D u=0.5, D-1=2 X 103$

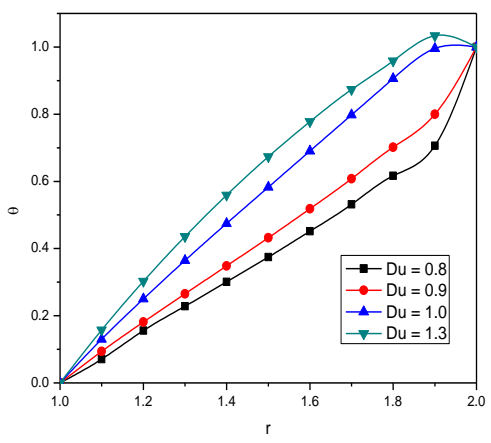

Fig.12. Variation of $\Theta$ with $D u, G=2 X 103, S c=1.3$, $S r=0.5, D-1=2 X 103, N=1$

The non-dimensional concentration $(\varphi)$ is shown in Figs. 13 to 18 for different values of the parametric values. It is found that the concentration gradually increases for all values. The variation of $\varphi$ with $G$ shows that it experiences an enhancement with increase in $G$ (Fig. 13). From Fig. 14 we notice that the actual concentration $\varphi$ increases with Darcy's parameter $\mathrm{D}^{-1}$. With respect to the variation of $\varphi$ with $\mathrm{Sc}$, we find that that lesser the molecular diffusitivity larger the actual concentration for all Sc $\leq 1.4$ and for further higher Sc $\geq 1.8$ it experiences a remarkable depreciation in the flow region (Fig. 15). Figure 16 shows that the actual concentration $\varphi$ increases with increase in Soret parameter $\mathrm{Sr} \leq 0.5$ and for further higher $\mathrm{Sr} \geq 0.8$ it experiences a remarkable depreciation in the flow region. From Fig. 17, we conclude that when the molecular buoyancy force dominates over the thermal buoyancy force it experiences depreciation irrespective of the directions of the buoyancy forces. The variation of $\varphi$ with Dufour parameter Du shows that the actual concentration $\varphi$ experiences depreciation in the flow region (Fig. 18).

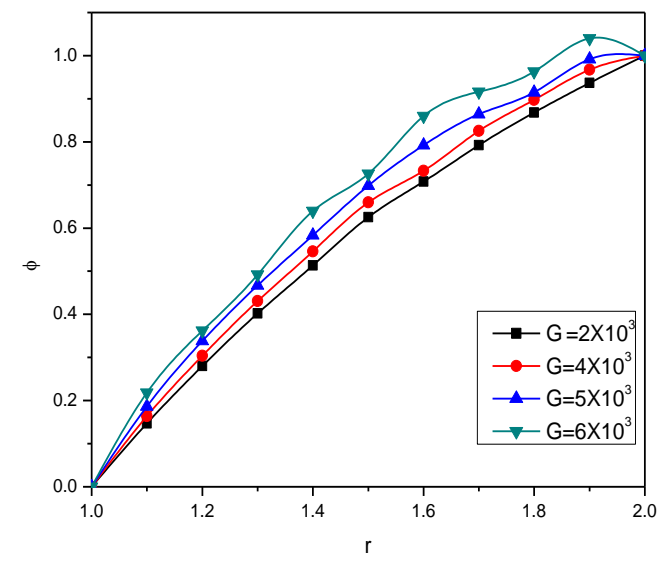

Fig.13. Variation of $\Phi$ with $G, D^{-1}=2 X 10^{3}, S c=1.3$, $S r=0.5, D u=0.5, N=1$.

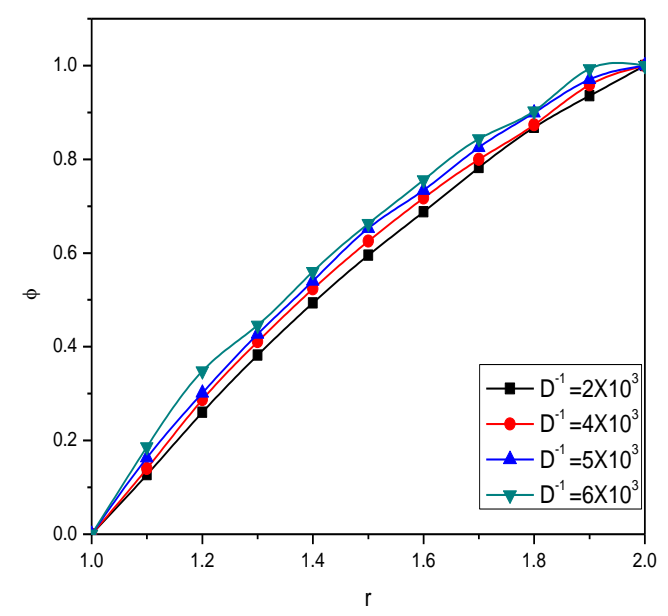

Fig.14. Variation of $\Phi$ with $D^{-1}, G=2 X 10^{3}, S c=1.3$, $S r=0.5, D u=0.5, N=1$.

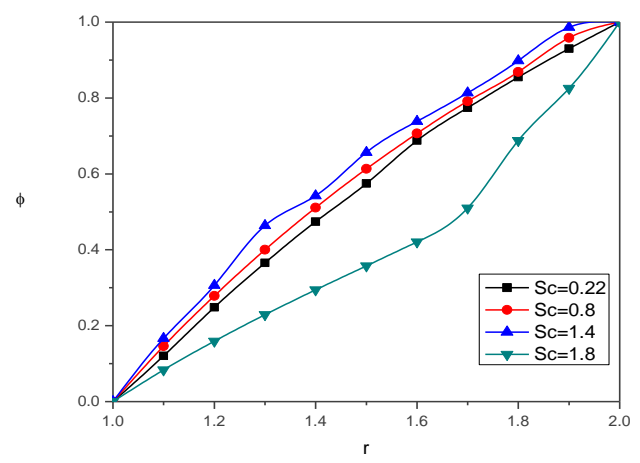

Fig.15. Variation of $\Phi$ with $S c, G=2 X 103, S r=0.5, D$ $1=2 \times 10^{3}, D u=0.5, N=1$ 


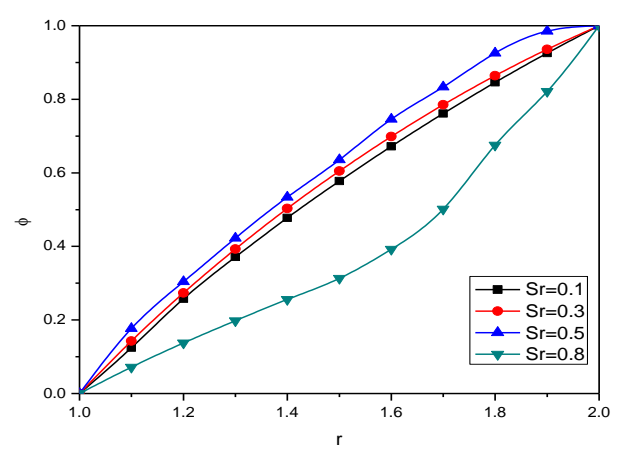

Fig.16. Variation of $\Phi$ with $\mathrm{Sr}, \mathrm{G}=2 \mathrm{X} 103, \mathrm{Sc}=1.3$, $\mathrm{Du}=0.5, \mathrm{~N}=1, \mathrm{D}-1=2 \mathrm{X} 10^{3}$

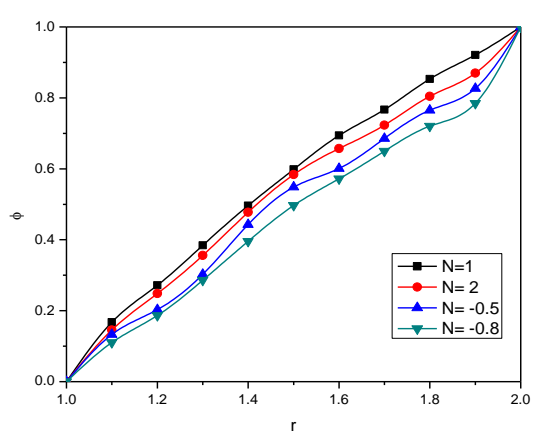

Fig.17. Variation of $\Phi$ with $\mathrm{N}, \mathrm{G}=2 \mathrm{X} 103, \mathrm{Sc}=1.3$, $\mathrm{Sr}=0.5, \mathrm{D}-1=2 \mathrm{X} 10^{3}$

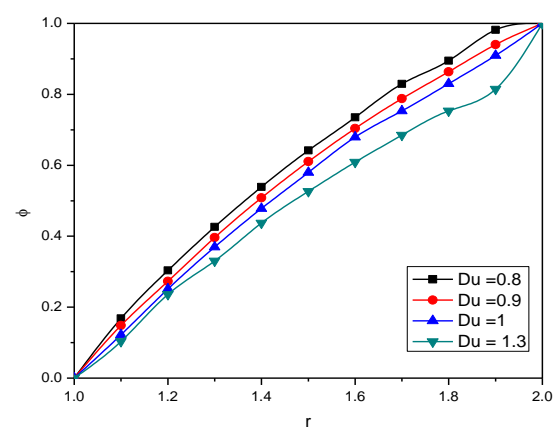

Fig.18. Variation of $\Phi$ with $\mathrm{Du}, \mathrm{G}=2 \mathrm{X} 10^{3}, \mathrm{Sc}=1.3$, $\mathrm{Sr}=0.5, \mathrm{Du}=0.5, \mathrm{~N}=1$.

\section{REFERENCES}

Agaral, R.S. and K.G. Upmanyu (1976). "Laminar free convection with and without heat sources in a circular pipe", Bull. Calcutta Math. Soc., 68, p 285.

Berman, A.S. (1958). J. Appl. Phys. 29, p 71.

Bhargava, R. and R.S. Agarwal (1979). "Fully developed free convection flow in a circular pipe", Indian J. Pure and Appl. Math. 10(3), p 357.
Cunningham, R.E and R.J. Williams (1980). Diffusion in gases and porous medium, Plenum, Newyork.

Gilpin, R.R. (1975). ”Cooling of a horizontal cylinder of water through its maximum density point at $4^{\circ} \mathrm{C}^{\prime}$, Int. J. Heat and Mass Transfer, 18, p 1307.

Goren, S.L. (1966). "On free convection in water at $4^{\circ} \mathrm{C} "$, Chem. Engg. Sci. 21, p 515.

Gulab Ram and R.S. Mishra (1977). Unsteady flow through magnetohydrodynamic porous media, Ind. J. Pure \& Appl. Math., V.8, p.637.

Kapoor, J.M. and S.C. Malik (1960). "Laminar flow in an annulus with rotating porous walls", Proc. Sixth Cong. Th. Appl. Mech. India, VI, p 125.

Mahapatra, J.R. (1973). "A note on the unsteady motion of a viscous conducting liquid between two porous concentric circular cylinders acted on by a radial magnetic field", Appl. Soc. Res. 27, p 274.

Padmavathi (2009). Finite element analysis of the convective heat transfer through a cylindrical annulus/Ducts in the presence of heat sources. $\mathrm{Ph} . \mathrm{D}$. Thesis, SK University, Anantapur, India.

Reddy (2009). The convective heat and mass transfer flow with thermo-diffusion effects in cylindrical annulus. Ph.D. Thesis, SK University, Anantapur, India.

Roy, S. (1972). Free convection in liquids under maximum density conditions, Indian J. Phys., 46, p 245.

Sarojamma, G. (1981). Magnetohydrodynamic convective flows. Ph.D. Thesis, SK University, Anantapur, India.

Siva Prasad, R.. Ph.D. Thesis, SK University, Anantapur, India. 